\title{
Search for Single Top Quark Production at HERA
}

\author{
H1 Collaboration
}

\begin{abstract}
A search for single top quark production is performed in the full $e^{ \pm} p$ data sample collected by the $\mathrm{H} 1$ experiment at HERA, corresponding to an integrated luminosity of $474 \mathrm{pb}^{-1}$. Decays of top quarks into a $b$ quark and a $W$ boson with subsequent leptonic or hadronic decay of the $W$ are investigated. A multivariate analysis is performed to discriminate top quark production from Standard Model background processes. An upper limit on the top quark production cross section via flavour changing neutral current processes $\sigma(e p \rightarrow e t X)<0.25 \mathrm{pb}$ is established at $95 \%$ CL. Limits on the anomalous coupling $\kappa_{t u \gamma}$ are derived.
\end{abstract}

Submitted to Phys. Lett. B 
F.D. Aaron ${ }^{5,49}$, M. Aldaya Martin ${ }^{11}$, C. Alexa $^{5}$, K. Alimujiang ${ }^{11}$, V. Andreev ${ }^{25}$,

B. Antunovic ${ }^{11}$, A. Asmone ${ }^{33}$, S. Backovic ${ }^{30}$, A. Baghdasaryan ${ }^{38}$, E. Barrelet ${ }^{29}$, W. Bartel ${ }^{11}$,

K. Begzsuren ${ }^{35}$, A. Belousov ${ }^{25}$, J.C. Bizot ${ }^{27}$, V. Boudry ${ }^{28}$, I. Bozovic-Jelisavcic ${ }^{2}$, J. Bracinik ${ }^{3}$,

G. Brandt ${ }^{11}$, M. Brinkmann ${ }^{12}$, V. Brisson ${ }^{27}$, D. Bruncko ${ }^{16}$, A. Bunyatyan ${ }^{13,38}$, G. Buschhorn ${ }^{26}$,

L. Bystritskaya ${ }^{24}$, A.J. Campbell ${ }^{11}$, K.B. Cantun Avila ${ }^{22}$, F. Cassol-Brunner ${ }^{21}$, K. Cerny ${ }^{32}$,

V. Cerny ${ }^{16,47}$, V. Chekelian ${ }^{26}$, A. Cholewa ${ }^{11}$, J.G. Contreras ${ }^{22}$, J.A. Coughlan ${ }^{6}$, G. Cozzika ${ }^{10}$,

J. Cvach ${ }^{31}$, J.B. Dainton ${ }^{18}$, K. Daum ${ }^{37,43}$, M. Deák ${ }^{11}$, Y. de Boer ${ }^{11}$, B. Delcourt ${ }^{27}$,

M. Del Degan ${ }^{40}$, J. Delvax ${ }^{4}$, A. De Roeck ${ }^{11,45}$, E.A. De Wolf ${ }^{4}$, C. Diaconu ${ }^{21}$, V. Dodonov ${ }^{13}$,

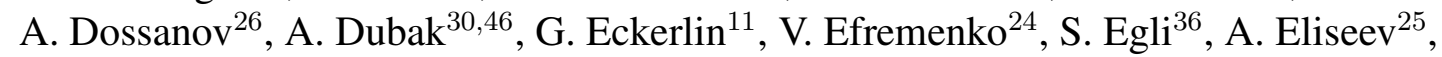

E. Elsen ${ }^{11}$, A. Falkiewicz ${ }^{7}$, L. Favart ${ }^{4}$, A. Fedotov ${ }^{24}$, R. Felst ${ }^{11}$, J. Feltesse ${ }^{10,48}$, J. Ferencei ${ }^{16}$,

D.-J. Fischer ${ }^{11}$, M. Fleischer ${ }^{11}$, A. Fomenko ${ }^{25}$, E. Gabathuler ${ }^{18}$, J. Gayler ${ }^{11}$, S. Ghazaryan ${ }^{38}$,

A. Glazov ${ }^{11}$, I. Glushkov ${ }^{39}$, L. Goerlich ${ }^{7}$, N. Gogitidze ${ }^{25}$, M. Gouzevitch ${ }^{11}$, C. Grab ${ }^{40}$,

T. Greenshaw ${ }^{18}$, B.R. Grell ${ }^{11}$, G. Grindhammer ${ }^{26}$, S. Habib ${ }^{12,50}$, D. Haidt ${ }^{11}$, C. Helebrant ${ }^{11}$,

R.C.W. Henderson ${ }^{17}$, E. Hennekemper ${ }^{15}$, H. Henschel ${ }^{39}$, M. Herbst ${ }^{15}$, G. Herrera ${ }^{23}$,

M. Hildebrandt ${ }^{36}$, K.H. Hiller ${ }^{39}$, D. Hoffmann ${ }^{21}$, R. Horisberger ${ }^{36}$, T. Hreus ${ }^{4,44}$, M. Jacquet ${ }^{27}$,

M.E. Janssen ${ }^{11}$, X. Janssen ${ }^{4}$, L. Jönsson ${ }^{20}$, A.W. Jung ${ }^{15}$, H. Jung ${ }^{11}$, M. Kapichine ${ }^{9}$, J. Katzy ${ }^{11}$,

I.R. Kenyon ${ }^{3}$, C. Kiesling ${ }^{26}$, M. Klein ${ }^{18}$, C. Kleinwort ${ }^{11}$, T. Kluge ${ }^{18}$, A. Knutsson ${ }^{11}$,

R. Kogler ${ }^{26}$, P. Kostka ${ }^{39}$, M. Kraemer ${ }^{11}$, K. Krastev ${ }^{11}$, J. Kretzschmar ${ }^{18}$, A. Kropivnitskaya ${ }^{24}$,

K. Krüger ${ }^{15}$, K. Kutak ${ }^{11}$, M.P.J. Landon ${ }^{19}$, W. Lange ${ }^{39}$, G. Laštovička-Medin ${ }^{30}$, P. Laycock ${ }^{18}$,

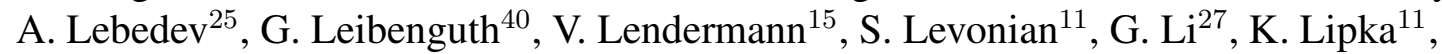

A. Liptaj ${ }^{26}$, B. List ${ }^{12}$, J. List ${ }^{11}$, N. Loktionova ${ }^{25}$, R. Lopez-Fernandez ${ }^{23}$, V. Lubimov ${ }^{24}$,

L. Lytkin ${ }^{13}$, A. Makankine ${ }^{9}$, E. Malinovski ${ }^{25}$, P. Marage ${ }^{4}$, Ll. Marti ${ }^{11}$, H.-U. Martyn ${ }^{1}$,

S.J. Maxfield ${ }^{18}$, A. Mehta ${ }^{18}$, A.B. Meyer ${ }^{11}$, H. Meyer ${ }^{11}$, H. Meyer ${ }^{37}$, J. Meyer ${ }^{11}$, V. Michels ${ }^{11}$,

S. Mikocki ${ }^{7}$, I. Milcewicz-Mika ${ }^{7}$, F. Moreau ${ }^{28}$, A. Morozov ${ }^{9}$, J.V. Morris ${ }^{6}$, M.U. Mozer ${ }^{4}$,

M. Mudrinic ${ }^{2}$, K. Müller ${ }^{41}$, P. Murín ${ }^{16,44}$, Th. Naumann ${ }^{39}$, P.R. Newman ${ }^{3}$, C. Niebuhr ${ }^{11}$,

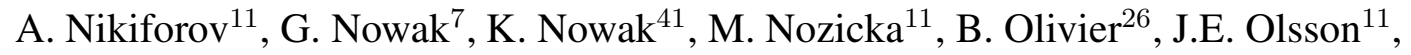

S. Osman ${ }^{20}$, D. Ozerov ${ }^{24}$, V. Palichik ${ }^{9}$, I. Panagoulias ${ }^{l, 11,42}$, M. Pandurovic ${ }^{2}$,

Th. Papadopoulou ${ }^{l, 11,42}$, C. Pascaud ${ }^{27}$, G.D. Patel ${ }^{18}$, O. Pejchal ${ }^{32}$, E. Perez $^{10,45}$,

A. Petrukhin ${ }^{24}$, I. Picuric ${ }^{30}$, S. Piec ${ }^{39}$, D. Pitzl ${ }^{11}$, R. Plačakyte ${ }^{11}$, B. Pokorny ${ }^{12}$, R. Polifka ${ }^{32}$,

B. Povh $^{13}$, T. Preda ${ }^{5}$, V. Radescu ${ }^{11}$, A.J. Rahmat ${ }^{18}$, N. Raicevic ${ }^{30}$, A. Raspiareza ${ }^{26}$,

T. Ravdandorj ${ }^{35}$, P. Reimer ${ }^{31}$, E. Rizvi ${ }^{19}$, P. Robmann ${ }^{41}$, B. Roland ${ }^{4}$, R. Roosen ${ }^{4}$,

A. Rostovtsev ${ }^{24}$, M. Rotaru ${ }^{5}$, J.E. Ruiz Tabasco ${ }^{22}$, Z. Rurikova ${ }^{11}$, S. Rusakov ${ }^{25}$, D. Š́lek ${ }^{32}$,

D.P.C. Sankey ${ }^{6}$, M. Sauter ${ }^{40}$, E. Sauvan ${ }^{21}$, S. Schmitt ${ }^{11}$, C. Schmitz ${ }^{41}$, L. Schoeffel ${ }^{10}$,

A. Schöning ${ }^{14}$, H.-C. Schultz-Coulon ${ }^{15}$, F. Sefkow ${ }^{11}$, R.N. Shaw-West ${ }^{3}$, L.N. Shtarkov ${ }^{25}$,

S. Shushkevich ${ }^{26}$, T. Sloan ${ }^{17}$, I. Smiljanic ${ }^{2}$, Y. Soloviev ${ }^{25}$, P. Sopicki ${ }^{7}$, D. South ${ }^{8}$, V. Spaskov ${ }^{9}$,

A. Specka ${ }^{28}$, Z. Staykova ${ }^{11}$, M. Steder ${ }^{11}$, B. Stella ${ }^{33}$, G. Stoicea ${ }^{5}$, U. Straumann ${ }^{41}$, D. Sunar ${ }^{4}$,

T. Sykora ${ }^{4}$, V. Tchoulakov ${ }^{9}$, G. Thompson ${ }^{19}$, P.D. Thompson ${ }^{3}$, T. Toll ${ }^{12}$, F. Tomasz ${ }^{16}$,

T.H. $\operatorname{Tran}^{27}$, D. Traynor ${ }^{19}$, T.N. Trinh ${ }^{21}$, P. Truöl ${ }^{41}$, I. Tsakov ${ }^{34}$, B. Tseepeldorj ${ }^{35,51}$, J. Turnau ${ }^{7}$,

K. Urban ${ }^{15}$, A. Valkárová ${ }^{32}$, C. Vallée ${ }^{21}$, P. Van Mechelen ${ }^{4}$, A. Vargas Trevino ${ }^{11}$, Y. Vazdik ${ }^{25}$,

S. Vinokurova ${ }^{11}$, V. Volchinski ${ }^{38}$, M. von den Driesch ${ }^{11}$, D. Wegener ${ }^{8}$, Ch. Wissing ${ }^{11}$,

E. Wünsch ${ }^{11}$, J. Žáček ${ }^{32}$, J. Zálešák ${ }^{31}$, Z. Zhang ${ }^{27}$, A. Zhokin ${ }^{24}$, T. Zimmermann ${ }^{40}$,

H. Zohrabyan ${ }^{38}$, F. Zomer ${ }^{27}$, and R. Zus ${ }^{5}$

${ }^{1}$ I. Physikalisches Institut der RWTH, Aachen, Germany ${ }^{a}$

${ }^{2}$ Vinca Institute of Nuclear Sciences, Belgrade, Serbia

${ }^{3}$ School of Physics and Astronomy, University of Birmingham, Birmingham, UK ${ }^{b}$ 
${ }^{4}$ Inter-University Institute for High Energies ULB-VUB, Brussels; Universiteit Antwerpen, Antwerpen; Belgium ${ }^{c}$

${ }^{5}$ National Institute for Physics and Nuclear Engineering (NIPNE), Bucharest, Romania

${ }^{6}$ Rutherford Appleton Laboratory, Chilton, Didcot, $U K^{b}$

${ }^{7}$ Institute for Nuclear Physics, Cracow, Poland ${ }^{d}$

${ }^{8}$ Institut für Physik, TU Dortmund, Dortmund, Germany ${ }^{a}$

${ }^{9}$ Joint Institute for Nuclear Research, Dubna, Russia

${ }^{10}$ CEA, DSM/Irfu, CE-Saclay, Gif-sur-Yvette, France

${ }^{11}$ DESY, Hamburg, Germany

${ }^{12}$ Institut für Experimentalphysik, Universität Hamburg, Hamburg, Germany ${ }^{a}$

${ }^{13}$ Max-Planck-Institut für Kernphysik, Heidelberg, Germany

${ }^{14}$ Physikalisches Institut, Universität Heidelberg, Heidelberg, Germany ${ }^{a}$

${ }^{15}$ Kirchhoff-Institut für Physik, Universität Heidelberg, Heidelberg, Germany ${ }^{a}$

${ }^{16}$ Institute of Experimental Physics, Slovak Academy of Sciences, Košice, Slovak Republic ${ }^{f}$

${ }^{17}$ Department of Physics, University of Lancaster, Lancaster, $U K^{b}$

${ }^{18}$ Department of Physics, University of Liverpool, Liverpool, $U K^{b}$

${ }^{19}$ Queen Mary and Westfield College, London, $U K^{b}$

${ }^{20}$ Physics Department, University of Lund, Lund, Sweden ${ }^{g}$

${ }^{21}$ CPPM, CNRS/IN2P3 - Univ. Mediterranee, Marseille, France

${ }^{22}$ Departamento de Fisica Aplicada, CINVESTAV, Mérida, Yucatán, México ${ }^{j}$

${ }^{23}$ Departamento de Fisica, CINVESTAV, México ${ }^{j}$

${ }^{24}$ Institute for Theoretical and Experimental Physics, Moscow, Russia ${ }^{k}$

${ }^{25}$ Lebedev Physical Institute, Moscow, Russia ${ }^{\text {}}$

${ }^{26}$ Max-Planck-Institut für Physik, München, Germany

${ }^{27}$ LAL, Univ Paris-Sud, CNRS/IN2P3, Orsay, France

${ }^{28}$ LLR, Ecole Polytechnique, IN2P3-CNRS, Palaiseau, France

${ }^{29}$ LPNHE, Universités Paris VI and VII, IN2P3-CNRS, Paris, France

${ }^{30}$ Faculty of Science, University of Montenegro, Podgorica, Montenegro ${ }^{e}$

${ }^{31}$ Institute of Physics, Academy of Sciences of the Czech Republic, Praha, Czech Republic ${ }^{h}$

${ }^{32}$ Faculty of Mathematics and Physics, Charles University, Praha, Czech Republic ${ }^{h}$

${ }^{33}$ Dipartimento di Fisica Università di Roma Tre and INFN Roma 3, Roma, Italy

${ }^{34}$ Institute for Nuclear Research and Nuclear Energy, Sofia, Bulgaria ${ }^{e}$

${ }^{35}$ Institute of Physics and Technology of the Mongolian Academy of Sciences, Ulaanbaatar, Mongolia

${ }^{36}$ Paul Scherrer Institut, Villigen, Switzerland

${ }^{37}$ Fachbereich C, Universität Wuppertal, Wuppertal, Germany

${ }^{38}$ Yerevan Physics Institute, Yerevan, Armenia

${ }^{39}$ DESY, Zeuthen, Germany

${ }^{40}$ Institut für Teilchenphysik, ETH, Zürich, Switzerland ${ }^{i}$

${ }^{41}$ Physik-Institut der Universität Zürich, Zürich, Switzerland ${ }^{i}$

${ }^{42}$ Also at Physics Department, National Technical University, Zografou Campus, GR-15773 Athens, Greece

${ }^{43}$ Also at Rechenzentrum, Universität Wuppertal, Wuppertal, Germany

${ }^{44}$ Also at University of P.J. Šafárik, Košice, Slovak Republic

${ }^{45}$ Also at CERN, Geneva, Switzerland 
${ }^{46}$ Also at Max-Planck-Institut für Physik, München, Germany

${ }^{47}$ Also at Comenius University, Bratislava, Slovak Republic

${ }^{48}$ Also at DESY and University Hamburg, Helmholtz Humboldt Research Award

${ }^{49}$ Also at Faculty of Physics, University of Bucharest, Bucharest, Romania

${ }^{50}$ Supported by a scholarship of the World Laboratory Björn Wiik Research Project

${ }^{51}$ Also at Ulaanbaatar University, Ulaanbaatar, Mongolia

${ }^{a}$ Supported by the Bundesministerium für Bildung und Forschung, FRG, under contract numbers $05 \mathrm{H1}$ 1GUA /1, $05 \mathrm{HI} \mathrm{1PAA/1,05} \mathrm{H1} \mathrm{1PAB} \mathrm{/9,} 05$ H1 1PEA /6, 05 H1 1VHA /7 and $05 \mathrm{HI} 1 \mathrm{VHB} / 5$

${ }^{b}$ Supported by the UK Science and Technology Facilities Council, and formerly by the UK Particle Physics and Astronomy Research Council

${ }^{c}$ Supported by FNRS-FWO-Vlaanderen, IISN-IIKW and IWT and by Interuniversity Attraction Poles Programme, Belgian Science Policy

${ }^{d}$ Partially Supported by Polish Ministry of Science and Higher Education, grant PBS/DESY/70/2006

${ }^{e}$ Supported by the Deutsche Forschungsgemeinschaft

${ }^{f}$ Supported by VEGA SR grant no. 2/7062/27

${ }^{g}$ Supported by the Swedish Natural Science Research Council

${ }^{h}$ Supported by the Ministry of Education of the Czech Republic under the projects LC527, INGO-1P05LA259 and MSM0021620859

${ }^{i}$ Supported by the Swiss National Science Foundation

${ }^{j}$ Supported by CONACYT, México, grant 48778-F

${ }^{k}$ Russian Foundation for Basic Research (RFBR), grant no 1329.2008.2

${ }^{l}$ This project is co-funded by the European Social Fund (75\%) and National Resources (25\%)

- (EPEAEK II) - PYTHAGORAS II 


\section{Introduction}

Top quarks are of particular interest in searches for new physics because their mass is close to the electroweak scale. In $e^{ \pm} p$ collisions at HERA the production of single top quarks is kinematically possible due to the large centre of mass energy of up to $\sqrt{s}=319 \mathrm{GeV}$. Within the Standard Model (SM) the production of top quarks at HERA is however strongly suppressed. Therefore the observation of single top quark production would be a clear indication of new physics. In several extensions of the SM the top quark is predicted to undergo flavour changing neutral current (FCNC) interactions, which may lead to a sizeable top quark production cross section at HERA [1,2].

A search for single top quark production is performed using the full $e^{ \pm} p$ data sample collected by the $\mathrm{H} 1$ experiment at HERA. The data correspond to an integrated luminosity of $474 \mathrm{pb}^{-1}$, including $36 \mathrm{pb}^{-1}$ of data taken at $\sqrt{s}=301 \mathrm{GeV}$. The analysis is inspired by and supersedes the previous $\mathrm{H} 1$ search for single top quark production based on an integrated luminosity of $118 \mathrm{pb}^{-1}$ [3]. Single top quark production is detected via the decay of the top quark $t \rightarrow b W$. In the case of leptonic decays of the $W$ boson, $W \rightarrow \ell \nu$, the signature is a charged lepton (electron or muon) and missing transverse momentum, accompanied by a hadronic final state $X$ with a high transverse momentum $\left(P_{T}\right)$ due to the $b$ quark. Events of this topology have been studied in a recently published analysis of events with isolated leptons and missing transverse momentum by $\mathrm{H} 1$ [4]. In the case of the hadronic $W$ boson decay channel, $W \rightarrow q \bar{q}$, the signature of single top quark production consists of three high $P_{T}$ jets with an invariant mass compatible with the top quark mass. A multivariate discriminant based on a neural network is used to differentiate top quark production from SM background. A possible contribution from a top quark signal is extracted using the method of fractional event counting [5].

\section{Single Top Quark Production}

The dominant process of SM single top quark production at HERA is via charged current (CC) production $e p \rightarrow \nu t b X$. The cross section for this process has been estimated [6] to be $\mathcal{O}(1) \mathrm{fb}$ and is thus not observable with the available integrated luminosity. Anomalous single top quark production within FCNC models, where the coupling of a top quark with an up-type quark $U$ via a photon is described by a coupling $\kappa_{t U \gamma}$, is illustrated in figure 1 . This process, as well as FCNC top quark interactions involving vector couplings to a $Z$ boson $V_{t U Z}$, can be described by an effective Lagrangian [7]:

$$
\mathcal{L}_{e f f}^{F C N C}=\sum_{U=u, c} \frac{e e_{U}}{2 \Lambda} \kappa_{t U \gamma} \bar{t} \sigma_{\mu \nu} A^{\mu \nu} U+\frac{g}{2 \cos \theta_{W}} V_{t U Z} \bar{t} \gamma_{\mu} U Z^{\mu}+\text { h.c. },
$$

where $\sigma_{\mu \nu}=(i / 2)\left[\gamma^{\mu}, \gamma^{\nu}\right], \theta_{W}$ is the Weinberg angle, $e$ and $g$ are the couplings to the electromagnetic and weak gauge groups with $U(1)$ and $S U(2)$ symmetries, respectively, $e_{U}$ is the electric charge of up-type quarks, $A^{\mu \nu}$ is the field strength tensor of the photon, $Z^{\mu}$ is the vector field of the $Z$ boson and $\Lambda$ is a scale parameter.

As the top quark mass is comparable to the ep centre of mass energy at HERA, the interacting parton in the proton must be at high Bjørken- $x$. Contributions from the charm quark are 


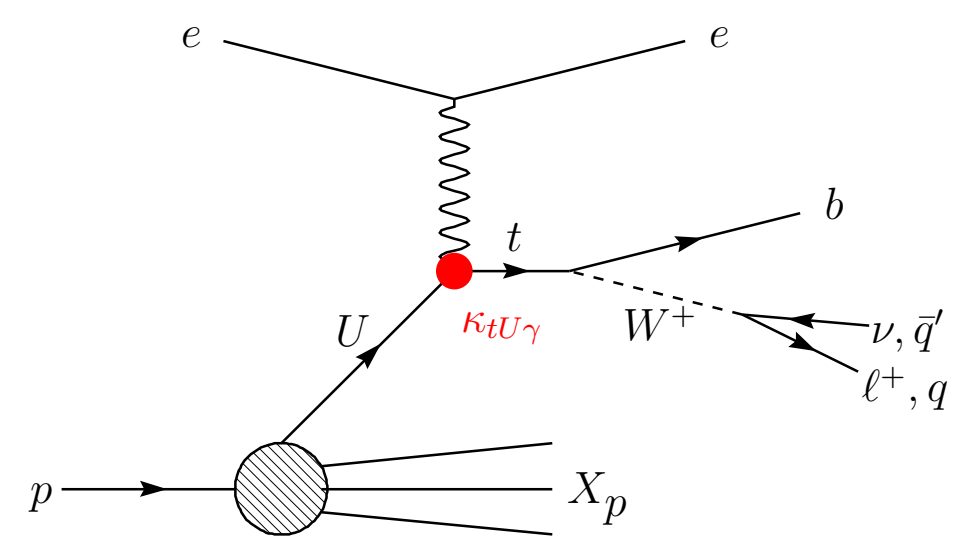

Figure 1: Anomalous single top quark production in $e p$ collisions via a FCNC coupling $\kappa_{t u \gamma}$.

therefore neglected $\left(\kappa_{t c \gamma} \equiv V_{t c Z} \equiv 0\right)$ since the $c$ quark density in the proton is low at high Bjørken- $x$. Similarly, the production of anti-top quarks is neglected, as this process involves sea anti-quarks in the initial state.

The simulation of an anomalous single top quark signal is done using the event generator ANOTOP [3], which uses the leading order (LO) matrix elements of the complete $e+q \rightarrow$ $e+t \rightarrow e+b+W \rightarrow e+b+f+\bar{f}^{\prime}$ process as obtained from the CompHEP program [8]. ANOTOP is used to calculate the production cross section and to study decays of top quarks in the $\mathrm{H} 1$ detector. Only top quark decays $t \rightarrow b W$ are considered as suggested by the strict limits on other possible top quark decays $[9,10]$.

The anomalous single top quark production cross section can then be parametrised as:

$$
\sigma(e p \rightarrow e t X, \sqrt{s})=c_{\gamma} \cdot \kappa_{t u \gamma}^{2}+c_{Z} \cdot V_{t u Z}^{2}+c_{\gamma Z} \cdot \kappa_{t u \gamma} \cdot V_{t u Z}
$$

The coefficients $c_{\gamma}$ and $c_{Z}$ are determined with ANOTOP at $\sqrt{s}=319 \mathrm{GeV}$. In this analysis $m_{\text {top }}$ is set to $175 \mathrm{GeV}$ and by convention the scale parameter $\Lambda$ is fixed to $m_{\text {top }}$. If $\Lambda \equiv m_{\text {top }}$ is reduced to $170 \mathrm{GeV}$, the cross section increases by $25 \%$, mainly due to an increased phase space for the production of top quarks. This mass range encompasses the current top quark mass determination of CDF and $\mathrm{D} \varnothing m_{\mathrm{top}}=172.4 \pm 1.2 \mathrm{GeV}$ [11] and corresponds to a coefficient range from $c_{\gamma}=7.53 \mathrm{pb}$ to $9.41 \mathrm{pb}$ and $c_{Z}=0.26 \mathrm{pb}$ to $0.32 \mathrm{pb}$. The values for $c_{\gamma}$ include next to leading order (NLO) corrections [12], which increase the LO cross section by $17 \%$. Including these NLO corrections results in an uncertainty related to the choice of the renormalisation and factorisation scales of about $5 \%$. Since the contribution of the $Z$ boson is small and no competitive sensitivity is expected, $Z$-exchange is neglected in this analysis and only the $\kappa_{t u \gamma}$ coupling is considered. The interference term with coefficient $c_{\gamma Z}$ contributes less than $1 \%$ of the total cross section and is therefore also neglected.

\section{Standard Model Background Processes}

The contributions from SM processes to the background in the leptonic decay channels were studied extensively in the analysis of events with isolated leptons and missing transverse mo- 
mentum [4] and are only briefly described here. The main SM background process is the production of single $W$ bosons. Further SM background processes may mimic the top quark signature through misidentification or mismeasurement. In the hadronic decay channel the SM background prediction is dominated by multi-jet events produced in photoproduction or neutral current (NC) deep-inelastic scattering (DIS).

Production of single $W$ bosons is modelled by the EPVEC generator [13]. NLO QCD corrections are taken into account by reweighting the generated events $[14,15]$. CC DIS events are simulated using the DJANGO [16] generator, which includes first order leptonic QED radiative corrections based on HERACLES [17]. The production of two or more jets in DJANGO is accounted for using the colour-dipole model [18]. The RAPGAP [19] event generator, which implements the Born, QCD Compton and Boson Gluon Fusion matrix elements, is used to model NC DIS events. Direct and resolved photoproduction of jets and prompt photon production are simulated using the PYTHIA [20] event generator. The simulation is based on Born level hard scattering matrix elements with radiative QED corrections. In RAPGAP and PYTHIA, jet production from higher order QCD radiation is simulated using leading logarithmic parton showers while hadronisation is modelled with Lund string fragmentation. The leading order MC prediction from NC DIS and photoproduction processes with two or more high transverse momentum jets is scaled by a factor of 1.2, to account for missing higher order QCD contributions in the MC generators [21,22]. Further small contributions to the SM background originate from lepton pair production, simulated using the GRAPE generator [23], and from photon wide angle bremsstrahlung, simulated in the WABGEN framework [24].

Generated signal and background events are passed through the GEANT [25] based simulation of the $\mathrm{H} 1$ detector, which takes into account the running conditions of the different data taking periods, and are reconstructed and analysed using the same program chain as for the data.

\section{Experimental Conditions}

A detailed description of the $\mathrm{H} 1$ experiment can be found in [26]. Only the detector components relevant to the present analysis are briefly described here. The origin of the H1 coordinate system is the nominal ep interaction point, with the direction of the proton beam defining the positive $z$-axis (forward region). Transverse momentum is measured in the $x y$-plane. The pseudorapidity $\eta$ is related to the polar angle $\theta$ by $\eta=-\ln \tan (\theta / 2)$. Tracking is provided by the central $\left(20^{\circ}<\theta<160^{\circ}\right)$ and forward $\left(7^{\circ}<\theta<25^{\circ}\right)$ tracking detectors. They are used to measure charged particle trajectories, to reconstruct the interaction vertex and to complement the measurement of hadronic energies. The tracking detectors are surrounded by a finely segmented Liquid Argon (LAr) calorimeter [27] that covers the polar angle range $4^{\circ}<\theta<154^{\circ}$. Electromagnetic shower energies are measured with a precision of $\sigma(E) / E=$ $12 \% / \sqrt{E / \mathrm{GeV}} \oplus 1 \%$ and hadronic energies with $\sigma(E) / E=50 \% / \sqrt{E / \mathrm{GeV}} \oplus 2 \%$, as measured in test beams $[28,29]$. In the backward region, energy measurements are provided by a lead/scintillating-fibre (SpaCal) calorimeter ${ }^{1}$ [30] covering the range $155^{\circ}<\theta<178^{\circ}$. The LAr calorimeter and inner tracking detectors are enclosed in a super-conducting magnetic coil

\footnotetext{
${ }^{1}$ This device was installed in 1995, replacing a lead-scintillator "sandwich" calorimeter [26].
} 
with a field strength of 1.16 T. From the curvature of charged particle trajectories in the magnetic field, the central tracking system provides transverse momentum measurements with a resolution of $\sigma_{P_{T}} / P_{T}=0.005 P_{T} / \mathrm{GeV} \oplus 0.015$ [31]. The return yoke of the magnetic coil is the outermost part of the detector and is equipped with streamer tubes forming the central muon detector $\left(4^{\circ}<\theta<171^{\circ}\right)$. In the forward region of the detector $\left(3^{\circ}<\theta<17^{\circ}\right)$ a set of drift chambers detects muons and measures their momenta using an iron toroidal magnet. The luminosity is determined from the rate of the Bethe-Heitler process $e p \rightarrow e p \gamma$, measured in a photon detector located close to the beam pipe at $z=-103 \mathrm{~m}$, in the backward direction.

The LAr calorimeter provides the main trigger for events in this analysis. The trigger efficiency is almost $100 \%$ for events with an electron with an energy above $10 \mathrm{GeV}$. Events with muons are triggered by an imbalance in transverse momentum measured in the calorimeter $P_{T}^{\text {calo }}$. The trigger efficiency is $\sim 60 \%$ for $P_{T}^{\text {calo }}=12 \mathrm{GeV}$, rising to $\simeq 98 \%$ for $P_{T}^{\text {calo }}>25 \mathrm{GeV}$. Events with jets have a trigger efficiency of nearly $100 \%$ for events with $P_{T}^{\text {jet }}>25 \mathrm{GeV}$ [32].

In order to remove background events induced by cosmic rays and other non-ep sources, the event vertex is required to be reconstructed within $\pm 35 \mathrm{~cm}$ in $z$ of the average interaction point. In addition, topological filters and timing vetoes are applied.

\section{Data Analysis}

\subsection{Particle Identification and Event Reconstruction}

Particle identification and hadronic final state reconstruction are described in detail in [4] and are only briefly summarised here. Electrons and photons are characterised [33] by compact and isolated electromagnetic clusters in the LAr calorimeter or SpaCal. Muon identification is based on a track in the inner tracking systems associated with signals in the muon detectors [34, 35]. Calorimeter energy deposits and tracks not previously identified as electron, photon or muon candidates are used to form combined cluster-track objects, from which the hadronic final state is reconstructed $[32,36]$. Jets are reconstructed from these combined cluster-track objects using an inclusive $k_{T}$ algorithm $[37,38]$ with a minimum transverse momentum of $4 \mathrm{GeV}$. The missing transverse momentum $P_{T}^{\text {miss }}$ in the event is derived from all detected particles and energy deposits in the event.

Strict isolation criteria are applied to electron and muon candidates. For electrons a track with a distance of closest approach (DCA) to the cluster of less than $12 \mathrm{~cm}$ is required to reject photons. The calorimetric energy measured within a distance in the pseudorapidity-azimuth $(\eta-\phi)$ plane $D=\sqrt{\Delta \eta^{2}+\Delta \phi^{2}}<0.5$ around the electron cluster is required to be below $3 \%$ of its energy. Furthermore, electrons are required to be isolated from jets by a distance $D$ (e; jet) $>1.0$ to any jet axis, and in the region $\theta>45^{\circ}$ by a distance $D$ (e; track) $>0.5$ from any track. A muon candidate may have no more than $5 \mathrm{GeV}$ energy deposited in a cylinder, centred on the muon track direction, of radius $25 \mathrm{~cm}$ and $50 \mathrm{~cm}$ in the electromagnetic and hadronic sections of the LAr calorimeter, respectively. Muon candidates are required to be separated from any jet by a distance $D(\mu$; jet $)>1.0$ and from any track by $D(\mu$; track $)>0.5$. 


\subsection{Systematic Uncertainties}

The following experimental systematic uncertainties are considered:

- The uncertainty on the electromagnetic energy scale varies depending on the polar angle from $0.7 \%$ to $2 \%$. The polar angle measurement uncertainty is $3 \mathrm{mrad}$ for electromagnetic clusters.

- The scale uncertainty on the transverse momentum of high $P_{T}$ muons is $2.5 \%$. The uncertainty on the muon polar angle measurement is $3 \mathrm{mrad}$.

- The hadronic energy scale is known within 1.5\% [39]. The uncertainty on the jet polar angle measurement is $10 \mathrm{mrad}$ [32].

- The uncertainty on the trigger efficiency is $3 \%$.

- The luminosity measurement has an uncertainty of $3 \%$.

The effect of the above systematic uncertainties on the MC expectation is determined by varying the experimental quantities by \pm 1 standard deviation in the MC samples and propagating these variations through the whole analysis.

Additional model uncertainties are attributed to the SM MC generators described in section 3. A theoretical uncertainty of $15 \%$ is used for the predicted contributions from EPVEC [14]. In the electron and muon channels the $\mathrm{CC}$ background contribution, which is modelled using DJANGO, is attributed a systematic error of $50 \%$ [4]. The contributions from background processes modelled using RAPGAP, PYTHIA, GRAPE and WABGEN are attributed $30 \%$ model uncertainties [4]. In the hadronic channel, the background processes with multi-jet final states modelled using RAPGAP and PYTHIA are attributed a 10\% model uncertainty determined from the comparison to data in an extended phase space.

\subsection{Electron and Muon Channels}

The search for single top quark production in the electron and muon channels is based on the selection described in [4]. Isolated electrons and muons with a transverse momentum $P_{T}^{\ell}>$ $10 \mathrm{GeV}$ in the polar angle range $5^{\circ}<\theta_{\ell}<140^{\circ}$ are selected in events with a missing transverse momentum $P_{T}^{\text {miss }}>12 \mathrm{GeV}$. In the electron channel, 39 events are found, compared to a SM prediction of $43.1 \pm 6.0$. In the muon channel, 14 events are observed, compared to a SM prediction of $11.0 \pm 1.8$. To estimate a potential top contribution to this sample, a top quark candidate is reconstructed from its decay products (lepton $\ell$, neutrino $\nu$ and $b$ quark), and the compatibility with single top quark production via FCNC is tested using a multivariate discriminant method.

The neutrino four-vector $P_{\nu}$ is reconstructed using the transverse and longitudinal momentum balance of the event. The transverse momentum of the neutrino is reconstructed by assuming:

$$
\vec{P}_{T}^{\nu} \equiv \vec{P}_{T}^{\text {miss }}
$$


If the scattered electron is detected in addition to the isolated lepton, the four-vector of the neutrino can be fully reconstructed by exploiting the energy and longitudinal momentum balance:

$$
\sum_{i}\left(E^{i}-P_{z}^{i}\right)+\left(E^{\nu}-P_{z}^{\nu}\right)=2 E_{e}^{0}=55.2 \mathrm{GeV}
$$

where the sum runs over all detected particles, $P_{z}$ is the momentum along the proton beam axis and $E_{e}^{0}$ is the electron beam energy. For events with more than one isolated electron, the electron with the lower transverse momentum is assumed to be the scattered electron [40]. If the scattered electron is not detected, the constraint $M^{\ell \nu} \simeq M_{W}=80.42 \mathrm{GeV}$ is applied. In the case that two physical solutions are obtained for $\left(E^{\nu}-P_{z}^{\nu}\right)$, the more probable solution according to the ANOTOP simulation is chosen [40]. A small fraction of events is removed in the case when the reconstruction algorithm finds no physical solution. In the electron channel, 38 events are selected after this neutrino reconstruction, while $39.7 \pm 5.6$ are expected from the SM. In the muon channel, 13 events are selected, while $10.7 \pm 1.6$ are expected from the SM. The selection efficiency for generated ANOTOP events at this stage is $49 \%$ (44\%) for the electron (muon) channel. The four-vector of the $b$-jet candidate is defined as the four-vector of the hadronic final state. The four-vector of the top quark candidate is reconstructed as the sum of the four-vectors of the isolated lepton, the neutrino and the $b$-jet candidate. This includes top quark decays where the $W$ boson decays via $W \rightarrow \tau \rightarrow e(\mu)$. Figure 2 shows the reconstructed top mass in the combined electron and muon channels. The data are in overall agreement with the SM prediction, while a slight excess of data events is observed in the top quark mass range.

In addition to the kinematic reconstruction of the top quark decay the lepton charge is also exploited, as the decay chain $t \rightarrow b W^{+} \rightarrow \ell \nu b$ produces only positively charged leptons. Well measured negatively charged leptons are rejected by requiring $q_{\ell} \cdot \frac{|\kappa|}{\delta \kappa}>-1.0$ where $q_{\ell}$ is the charge of the lepton, $|\kappa|$ is the curvature of the track associated to the lepton and $\delta \kappa$ is the error on the curvature. This requirement is only applied in the central region where the charge determination by the tracking detectors is reliable. Table 1 summarises the event yield in the electron and muon channels for the resulting top preselection. In the electron channel 30 events are selected, while $31.5 \pm 4.0$ are expected from the SM. In the muon channel, eight events are selected, while $8.0 \pm 0.9$ are expected from the SM.

The following observables are then used to further discriminate single top quark production via FCNC against SM background:

- $P_{T}^{b}$, the transverse momentum of the $b$-jet candidate;

- $M^{\ell \nu b}$, the invariant mass of the reconstructed top quark;

- $\theta_{W^{*}}^{\ell}$, the $W$ decay angle calculated as the angle between the lepton momentum in the $W$ rest frame and the $W$ direction in the top quark rest frame.

Distributions of these observables are shown in figures 3 (a-c) and 4 (a-c) for the electron and muon channels, respectively. Good overall agreement of the data with the SM expectation is seen in all distributions. For the discrimination of top and background events, a multivariate discriminator based on a multilayer perceptron (MLP) neural network was trained on the signal and background simulations. Figures 3 (d) and 4 (d) show the MLP discriminator output 
distributions for the electron and muon channels, respectively. A good agreement is observed between data and SM expectation, with some events visible in the signal region.

Results from the multivariate analysis are cross checked with a cut-based top selection requiring for the top preselected events in addition $P_{T}^{b}>30 \mathrm{GeV}$ and $M^{\ell \nu b}>140 \mathrm{GeV}$. The resulting event yields are also shown in table 1 . In this selection five events are selected in the electron channel, while $3.2 \pm 0.4$ are expected from the SM. In the muon channel, four events are selected, while $2.1 \pm 0.3$ are expected from the SM. This sample includes all five top-like events found in the previous analysis [3]. If the charge requirement is not applied, the corresponding event yields are seven selected events for an expectation of $4.1 \pm 0.7$ in the electron channel, and six events for $2.8 \pm 0.4$ expected in the muon channel.

\subsection{Hadronic Channel}

The hadronic decay of the $W$ boson from the top decay $t \rightarrow b W \rightarrow b q \overline{q^{\prime}}$ leads to events with at least three jets with high transverse momenta. SM background arises mainly from multi-jet events in photoproduction or NC DIS. A top preselection is defined by selecting events with at least three jets in the pseudorapidity range $-0.5<\eta^{\text {jet }}<2.5$. The jets are ordered by the magnitude of their transverse momenta and only events with $P_{T}^{\text {jet1 }}>40 \mathrm{GeV}, P_{T}^{\text {jet} 2}>30 \mathrm{GeV}$ and $P_{T}^{\text {jet3 }}>15 \mathrm{GeV}$ are selected. A cut on the scalar sum of the jet transverse momenta $\sum P_{T}^{\mathrm{jet}}>110 \mathrm{GeV}$ is also applied. In addition, one of the jet pairings must yield an invariant mass between $65 \mathrm{GeV}$ and $95 \mathrm{GeV}$, corresponding to a window around the nominal $W$ mass with a width of twice the mass resolution obtained for hadronic $W$ decays [40]. The remaining jet that is not used to form the $W$ candidate is considered to originate from the $b$ quark and is required to have a minimum $P_{T}>25 \mathrm{GeV}$. The yield for this top preselection is given in table 1. A total of 404 events are selected, compared to a SM prediction of $388 \pm 32$ events. The selection efficiency for generated ANOTOP events is $42 \%$ at this stage. Analogously to the leptonic channel observables for the discrimination of the top quark signal from the QCD background are chosen:

- $P_{T}^{b}$, the transverse momentum of the $b$-jet candidate;

- $M^{\text {jets }}$, the invariant mass of all jets in the event corresponding to the mass of the top quark for signal events;

- $\theta_{W^{*}}^{q}$, the $W$ decay angle defined as the angle in the $W$ rest frame between the lowest $P_{T}$ jet of the two jets associated to the $W$ decay and the $W$ direction in the top quark rest frame.

Distributions of these three observables are shown in figure 5 (a-c), compared to the SM expectation and the simulated top signal. Good agreement between the data and the SM simulation is seen for all three distributions. For the discrimination of top and background events, an MLP discriminator was trained on the signal and background simulations. Figure 5 (d) shows the discriminator output distribution for the signal and background simulations. Also shown are the data events as classified by the discriminator and a good agreement is observed between data 
and SM expectation. This distribution is used to extract a possible signal, as described in the next section.

A cut-based top selection is also performed in the hadronic channel. The transverse momentum of the $b$-jet candidate has to fulfill $P_{T}^{b}>40 \mathrm{GeV}$ and the reconstructed top quark mass $150<M^{\text {jets }}<210 \mathrm{GeV}$. The number of candidate events selected is 128 , compared with $123 \pm 13$ events expected from SM processes.

\section{Results}

The discriminant distributions observed in all three channels are in correspondence with the SM expectation as shown in figures 3 (d), 4 (d) and 5 (d). Since no significant indication for the production of single top quarks is observed, upper limits on the cross section are derived using the method of fractional event counting which takes efficiencies, statistical and systematic uncertainties into account [5]. This method is cross-checked using a modified frequentist approach [41] which is found to yield similar results. Also expected cross section limits are determined by assuming that a top quark signal is a fluctuation of Poisson distributed SM background. An ensemble of toy experiments with this hypothesis is conducted using the background prediction and calculating a cross section limit at $95 \% \mathrm{CL}$ for each toy experiment. The mean of the resulting distribution is reported as the expected cross section limit at 95\% CL.

For all channels combined an upper bound on the cross section for single top quark production via FCNC is obtained at the 95\% CL:

$$
\sigma(e p \rightarrow e t X, \sqrt{s}=319 \mathrm{GeV})<0.25 \mathrm{pb} .
$$

This cross section limit is reported for $\sqrt{s}=319 \mathrm{GeV}$, taking into account the ratio 0.70 of the predicted signal cross sections at $\sqrt{s}=301 \mathrm{GeV}$ and $319 \mathrm{GeV}$ [12]. Table 2 lists the observed and expected cross section limits for the individual channels, the combined leptonic channels, and all channels combined.

The top-like events observed in the data at high values of the discriminant lead to observed limits that are weaker than the expected limits. This difference corresponds to the slightly higher observed event yields from the cut-based top selection compared to the predictions. Extracting a production cross section from the discriminant distributions gives for all channels combined $\sigma(e p \rightarrow e t X, \sqrt{s}=319 \mathrm{GeV})=0.11 \pm 0.07 \mathrm{pb}$. This value is compatible with zero within two standard deviations.

The limits on the cross section are converted to limits at $95 \%$ CL on the anomalous FCNC coupling $\kappa_{t u \gamma}$ using equation 1 . This results in an upper limit on $\kappa_{t u \gamma}<0.16-0.18$ for the parameter range $\Lambda \equiv m_{\text {top }} \equiv 170-175 \mathrm{GeV}$, respectively. Figure 6 shows current limits from experiments in the $\kappa_{t u \gamma}-V_{t u Z}$ plane. The limits on branching fractions of anomalous decays of top quarks obtained by the CDF experiment at Tevatron $[9,10]$ are converted to limits on couplings using the conventions described in section 2. The limit on the branching fraction $\mathcal{B}(t \rightarrow q Z)<3.7 \%$ at $95 \% \mathrm{CL}$ reported by $\mathrm{CDF}$ [9] corresponds to the strictest limit on the $V_{t u Z}$ coupling. For ease of comparison, the $\mathrm{H} 1$ limit on the coupling $\kappa_{t u \gamma}$ is also converted to a limit on the branching fraction $\mathcal{B}(t \rightarrow u \gamma)<0.64 \%$, also shown in table 2 . The LEP 
experiments have also searched for anomalous single top quark production [42]. The limit obtained by the ZEUS experiment [43] using $130 \mathrm{pb}^{-1}$ of data is similar to the $\mathrm{H} 1$ bound based on the full HERA data, mainly reflecting the slight excess of top-like events observed by $\mathrm{H} 1$, but not by ZEUS. The present analysis explores a domain not covered by other colliders.

\section{Summary}

A search for single top quark production via FCNC at HERA is performed using the full $e^{ \pm} p$ data sample, corresponding to an integrated luminosity of $474 \mathrm{pb}^{-1}$. The search is based on a sample of events with isolated leptons and missing transverse momentum and a sample of multijet events. No evidence for single top quark production is observed. Within FCNC models the top quark production cross section is extracted from multivariate discriminator distributions using the method of fractional event counting. An upper limit at $95 \% \mathrm{CL}$ on the production cross section:

$$
\sigma(e p \rightarrow e t X, \sqrt{s}=319 \mathrm{GeV})<0.25 \mathrm{pb}
$$

is established. This result is translated into a limit on the anomalous coupling $\kappa_{t u \gamma}$ at $95 \% \mathrm{CL}$ :

$$
\kappa_{t u \gamma}<0.18
$$

if the scale of the new physics $\Lambda \equiv m_{\text {top }} \equiv 175 \mathrm{GeV}$. This corresponds to a limit on the branching ratio $\mathcal{B}(t \rightarrow u \gamma)<0.64 \%$.

\section{Acknowledgements}

We are grateful to the HERA machine group whose outstanding efforts have made this experiment possible. We thank the engineers and technicians for their work in constructing and maintaining the $\mathrm{H} 1$ detector, our funding agencies for financial support, the DESY technical staff for continual assistance and the DESY directorate for support and for the hospitality which they extend to the non-DESY members of the collaboration.

\section{References}

[1] H. Fritzsch and D. Holtmannspötter, Phys. Lett. B 457 (1999) 186 [hep-ph/9901411].

[2] J. A. Aguilar-Saavedra, Acta Phys. Polon. B 35 (2004) 2695 [hep-ph/0409342].

[3] A. Aktas et al. [H1 Collaboration], Eur. Phys. J. C 33 (2004) 9 [hep-ex/0310032].

[4] F. D. Aaron et al. [H1 Collaboration], arXiv:0901.0488.

[5] P. Bock, JHEP 0701 (2007) 080 [hep-ex/0405072].

[6] T. Stelzer, Z. Sullivan and S. Willenbrock, Phys. Rev. D 56 (1997) 5919 [hep-ph/9705398]. 
[7] T. Han and J. L. Hewett, Phys. Rev. D 60 (1999) 074015 [hep-ph/9811237].

[8] E. E. Boos et al., SNUTP-94-116 [hep-ph/9503280];

E. E. Boos et al., Proceedings of the Xth Int. Workshop on High Energy Physics and Quantum Field Theory, QFTHEP-95, Moscow (1995), Eds. B. Levtchenko and V. Savrin, p 101.

[9] T. Aaltonen et al. [CDF Collaboration], Phys. Rev. Lett. 101 (2008) 192002 [arXiv:0805.2109].

[10] F. Abe et al. [CDF Collaboration], Phys. Rev. Lett. 80 (1998) 2525.

[11] Tevatron Electroweak Working Group [CDF Collaboration and D $\varnothing$ Collaboration], arXiv:0808.1089.

[12] A. Belyaev and N. Kidonakis, Phys. Rev. D 65 (2002) 037501 [hep-ph/0102072].

[13] U. Baur, J. A. Vermaseren and D. Zeppenfeld, Nucl. Phys. B 375 (1992) 3.

[14] K. P. O. Diener, C. Schwanenberger and M. Spira, Eur. Phys. J. C 25 (2002) 405 [hep-ph/0203269];

P. Nason, R. Rückl and M. Spira, J. Phys. G 25 (1999) 1434 [hep-ph/9902296];

M. Spira, DESY-99-060 [hep-ph/9905469].

[15] K. P. O. Diener, C. Schwanenberger and M. Spira, hep-ex/0302040.

[16] G. A. Schuler and H. Spiesberger, DJANGOH 1.4, Comput. Phys. Commun. 81 (1994) 381.

[17] A. Kwiatkowski, H. Spiesberger and H. J. Möhring, Heracles 1.0, Comput. Phys. Commun. 69 (1992) 155.

[18] L. Lönnblad, ARIADNE 4, Comput. Phys. Commun. 71 (1992) 15.

[19] H. Jung, RAPGAP 3.1, Comput. Phys. Commun. 86 (1995) 147.

[20] T. Sjostrand et. al, PYTHIA 6.1, Comput. Phys. Commun. 135 (2001) 238 [hep-ph/0010017].

[21] C. Adloff et al. [H1 Collaboration], Eur. Phys. J. C 25 (2002) 13 [hep-ex/0201006].

[22] F. D. Aaron et al. [H1 Collaboration], arXiv:0901.0507.

[23] T. Abe, GRAPE-Dilepton 1.1, Comput. Phys. Commun. 136 (2001) 126 [hep$\mathrm{ph} / 0012029]$.

[24] C. Berger and P. Kandel, "A new generator for wide angle bremsstrahlung," Proceedings of the Workshop "Monte Carlo Generators for HERA Physics", eds. A. T. Doyle, G. Grindhammer, G. Ingelman and H. Jung, DESY (1998), DESY-PROC-1999-02, 596.

[25] R. Brun et al., “GEANT3”, CERN-DD/EE/84-1. 
[26] I. Abt et al. [H1 Collaboration], Nucl. Instrum. Meth. A 386 (1997) 310;

I. Abt et al. [H1 Collaboration], Nucl. Instrum. Meth. A 386 (1997) 348.

[27] B. Andrieu et al. [H1 Calorimeter Group Collaboration], Nucl. Instrum. Meth. A 336 (1993) 460.

[28] B. Andrieu et al. [H1 Calorimeter Group Collaboration], Nucl. Instrum. Meth. A 336 (1993) 499.

[29] B. Andrieu et al. [H1 Calorimeter Group Collaboration], Nucl. Instrum. Meth. A 350 (1994) 57.

[30] R. D. Appuhn et al. [H1 SPACAL Group Collaboration], Nucl. Instrum. Meth. A 386 (1997) 397.

[31] C. Kleinwort [H1 Collaboration], Proceedings of the Workshop "First LHC Detector Alignment Workshop”, eds. S. Blusk et al., CERN (2006), CERN-2007-04.

[32] M. Peez, "Recherche de déviations au Model Standard dans les processus de grande énergie transverse sur le collisionneur électron - proton HERA", Ph.D. thesis, Université de Lyon (2003), DESY-THESIS-2003-023

(available at http://www-h1.desy.de/psfiles/theses/).

[33] P. Bruel, "Recherche d'interactions au-delà du Modèle Standard à HERA", Ph.D. Thesis, Université Paris XI (1998)

(available at http://www-h1.desy.de/psfiles/theses/).

[34] A. Aktas et al. [H1 Collaboration], Phys. Lett. B 583 (2004) 28 [hep-ex/0311015].

[35] F. D. Aaron et al. [H1 Collaboration], Phys. Lett. B 668 (2008) 268 [arXiv:0806.3987].

[36] B. Portheault, "Première mesure des sections efficaces de courant chargé et neutre avec le faisceau de positrons polarisé à HERA II et analyses QCD-électrofaibles", Ph.D. thesis, Université Paris XI (2005), LAL-05-05

(available at http://www-h1.desy.de/psfiles/theses/).

[37] S. D. Ellis and D. E. Soper, Phys. Rev. D 48 (1993) 3160 [hep-ph/9305266].

[38] S. Catani et al., Nucl. Phys. B 406 (1993) 187.

[39] M. Gouzevitch, "Mesure de la constante de couplage forte $\alpha_{s}$ avec les jets hadroniques en Diffusion Inélastique Profonde", Ph.D. thesis, Ecole Polytechnique, Palaiseau (2008) (available at http://www-h1.desy.de/psfiles/theses/).

[40] J. Dingfelder, "Search for anomalous production of single top quarks with the H1 experiment at HERA", Ph.D. thesis, University of Heidelberg (2003)

(available at http://www-h1.desy.de/psfiles/theses/).

[41] T. Junk, Nucl. Instrum. Meth. A 434 (1999) 435 [hep-ex/9902006]. 
[42] P. Achard et al. [L3 Collaboration], Phys. Lett. B 549 (2002) 290 [hep-ex/0210041]; J. Abdallah et al. [DELPHI Collaboration], Phys. Lett. B 590 (2004) 21 [hep-ex/0404014]; A. Heister et al. [ALEPH Collaboration], Phys. Lett. B 543 (2002) 173 [hep-ex/0206070]; G. Abbiendi et al. [OPAL Collaboration], Phys. Lett. B 521 (2001) 181 [hep-ex/0110009].

[43] S. Chekanov et al. [ZEUS Collaboration], Phys. Lett. B 559 (2003) 153 [hep-ex/0302010]; S. Chekanov et al. [ZEUS Collaboration], DESY-03-188. 


\begin{tabular}{|c|c|c|c|c|}
\hline \multicolumn{5}{|c|}{ H1 Search for Single Top Production at HERA $\left(e^{ \pm} p, 474 \mathrm{pb}^{-1}\right)$} \\
\hline \multicolumn{2}{|r|}{ Electron Channel } & Data & Standard Model & Top Efficiency \\
\hline \multirow{3}{*}{ 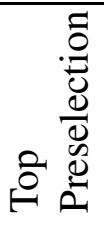 } & Isolated Leptons & 39 & $43.1 \pm 6.0$ & $49 \%$ \\
\hline & $\nu$ Reconstruction & 38 & $39.7 \pm 5.6$ & $49 \%$ \\
\hline & Cut on Lepton Charge & 30 & $31.5 \pm 4.0$ & $49 \%$ \\
\hline \multicolumn{2}{|r|}{ Cut-based Top Selection } & 5 & $3.2 \pm 0.4$ & $40 \%$ \\
\hline \multicolumn{2}{|r|}{ Muon Channel } & Data & Standard Model & Top Efficiency \\
\hline \multirow{3}{*}{ 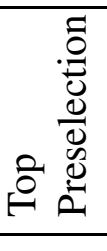 } & Isolated Leptons & 14 & $11.0 \pm 1.8$ & $44 \%$ \\
\hline & $\nu$ Reconstruction & 13 & $10.7 \pm 1.6$ & $44 \%$ \\
\hline & Cut on Lepton Charge & 8 & $8.0 \pm 0.9$ & $44 \%$ \\
\hline \multicolumn{2}{|r|}{ Cut-based Top Selection } & 4 & $2.1 \pm 0.3$ & $36 \%$ \\
\hline \multicolumn{2}{|r|}{ Hadron Channel } & Data & Standard Model & Top Efficiency \\
\hline \multicolumn{2}{|r|}{ Top Preselection } & 404 & $388 \pm 32$ & $42 \%$ \\
\hline \multicolumn{2}{|r|}{ Cut-based Top Selection } & 128 & $123 \pm 13$ & $33 \%$ \\
\hline
\end{tabular}

Table 1: Observed and predicted numbers of events in the electron, muon and hadronic channels for the steps of the top preselection and in the cut-based top selection. The top signal efficiency is estimated using ANOTOP. The quoted errors take into account all statistical and systematic uncertainties. 


\begin{tabular}{|c|cc|c|c|}
\hline \multicolumn{6}{|c|}{ H1 Search for Single Top Production $\left(e^{ \pm} \boldsymbol{p}, \mathbf{4 7 4} \mathbf{p b}^{-\mathbf{1}}\right)$} \\
\hline \hline \multirow{2}{*}{ Channel } & \multicolumn{4}{|c|}{ Upper Limit at $95 \% \mathrm{CL}$} \\
\cline { 2 - 5 } & $\sigma(e p \rightarrow t X, \sqrt{s}=319 \mathrm{GeV})$ & $\kappa_{t u \gamma}$ & $\mathcal{B}(t \rightarrow u \gamma)$ \\
& Observed $[\mathrm{pb}]$ & Expected $[\mathrm{pb}]$ & & {$[\%]$} \\
\hline Electron & 0.40 & 0.24 & $0.21-0.23$ & $0.82-1.02$ \\
Muon & 0.30 & 0.22 & $0.18-0.20$ & $0.61-0.76$ \\
Electron+Muon & 0.27 & 0.15 & $0.17-0.19$ & $0.55-0.69$ \\
Hadronic & 0.42 & 0.27 & $0.21-0.24$ & $0.86-1.07$ \\
Combined & 0.25 & 0.12 & $0.16-0.18$ & $0.51-0.64$ \\
\hline
\end{tabular}

Table 2: Observed and expected upper limits at 95\% CL on the single top production cross section at $\sqrt{s}=319 \mathrm{GeV}$ in the electron, muon, hadronic and combined channels. Also shown are upper limits on the anomalous coupling $\kappa_{t u \gamma}$ and on the branching fraction $\mathcal{B}(t \rightarrow u \gamma)$. These limits are shown for $\Lambda \equiv m_{\text {top }} \equiv 170-175 \mathrm{GeV}$. 
Search for Single Top Quarks at HERA (474 $\left.\mathrm{pb}^{-1}\right)$

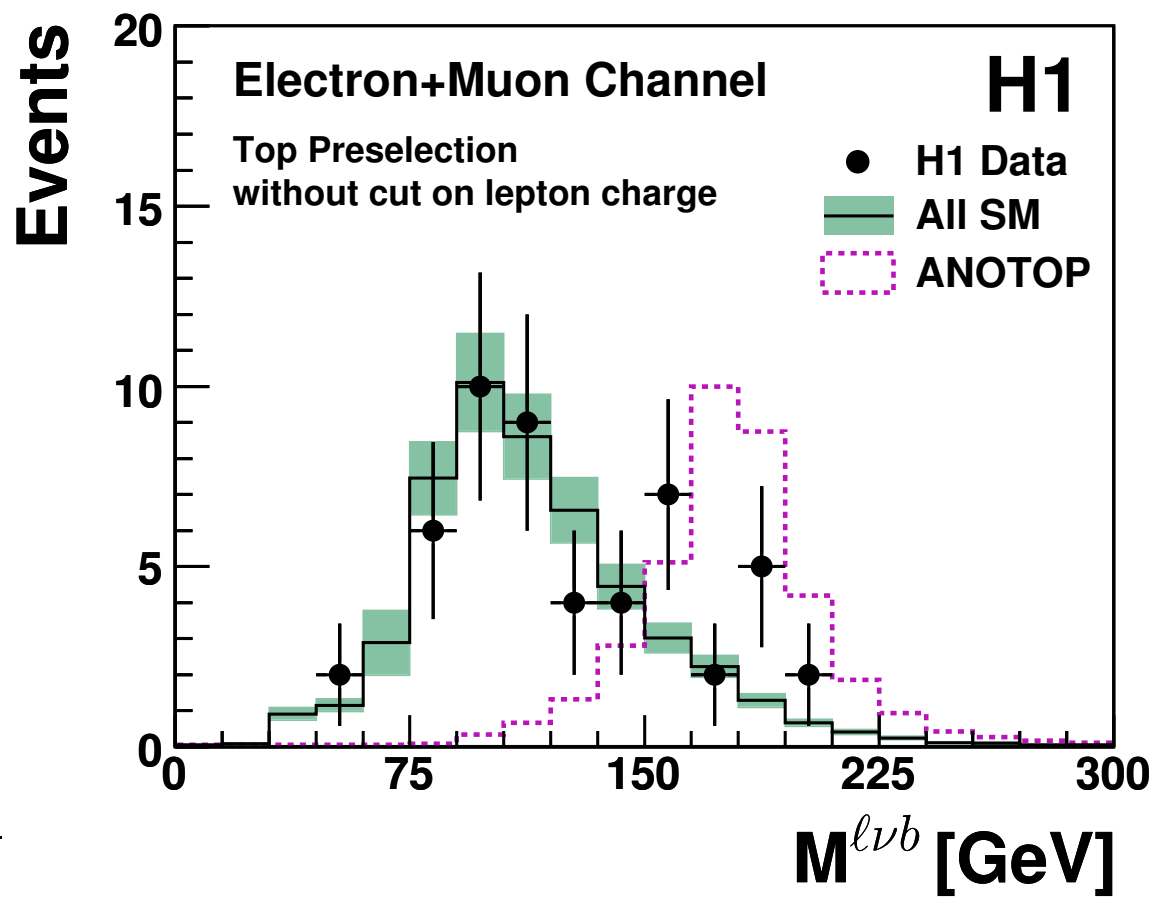

Figure 2: Distribution of the reconstructed top mass $M^{\ell \nu b}$ in the electron and muon channels after $\nu$ reconstruction but before the cut on the lepton charge. The data are shown as points, the total SM expectation as the open histogram with systematic and statistical uncertainties added in quadrature (shaded band). Also shown is the ANOTOP prediction with arbitrary normalisation (dashed histogram). 


\section{Search for Single Top Quark Production at HERA (474 pb-1)}
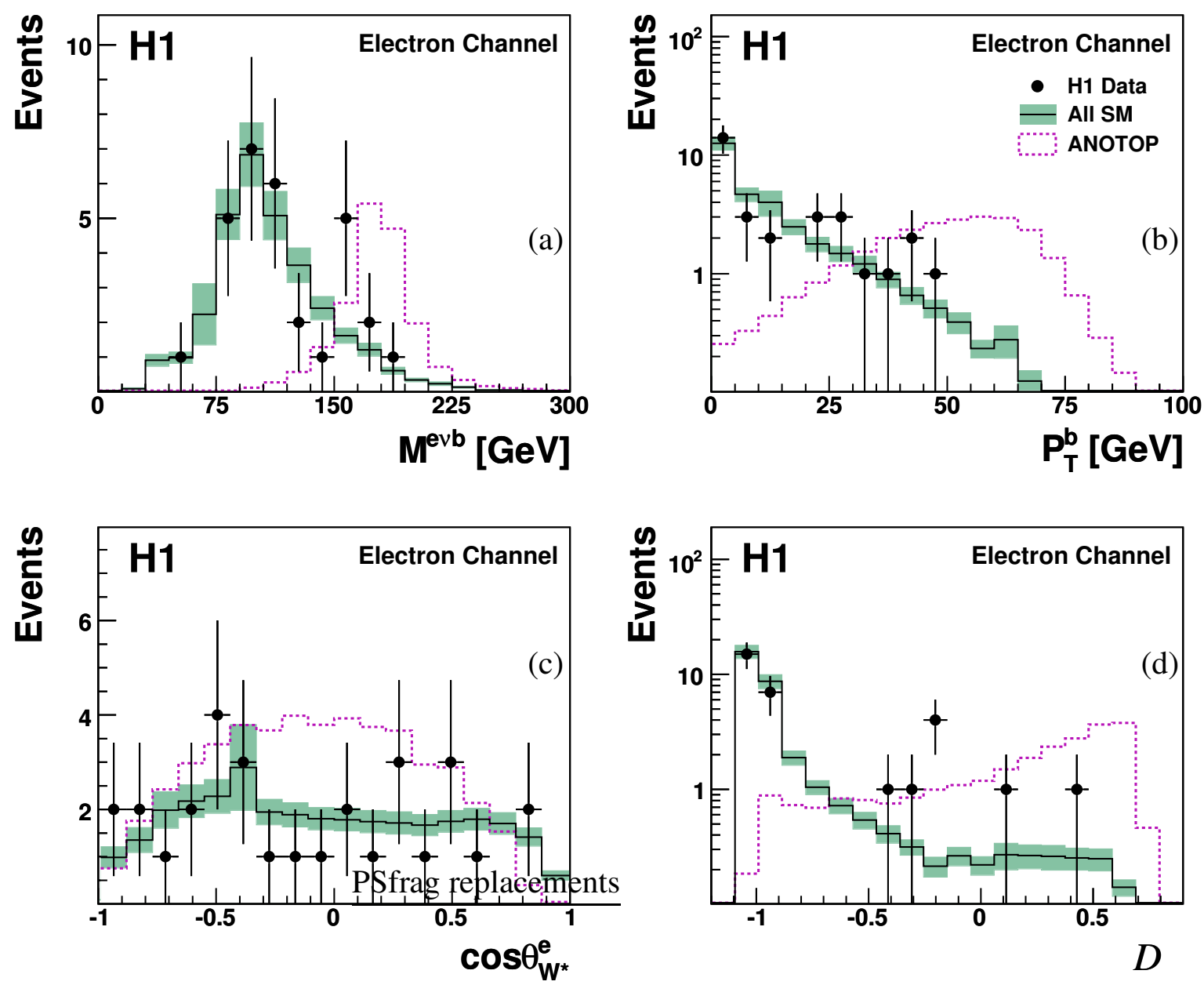

Figure 3: Distributions of observables in the electron channel used to differentiate single top production from SM background processes in the top preselection. Shown are the reconstructed top mass $M^{e \nu b}$ (a), the transverse momentum of the reconstructed $b$-jet candidate $P_{T}^{b}$ (b), the $W$ decay angle $\cos \theta_{W^{*}}^{e}(\mathrm{c})$, and the combination of these observables into a MLP-based discriminant $D(\mathrm{~d})$. The data are shown as points, the total SM expectation as the open histogram with systematic and statistical uncertainties added in quadrature (shaded band). Also shown is the signal prediction with arbitrary normalisation (dashed histogram). 


\section{Search for Single Top Quark Production at HERA (474 $\left.\mathrm{pb}^{-1}\right)$}
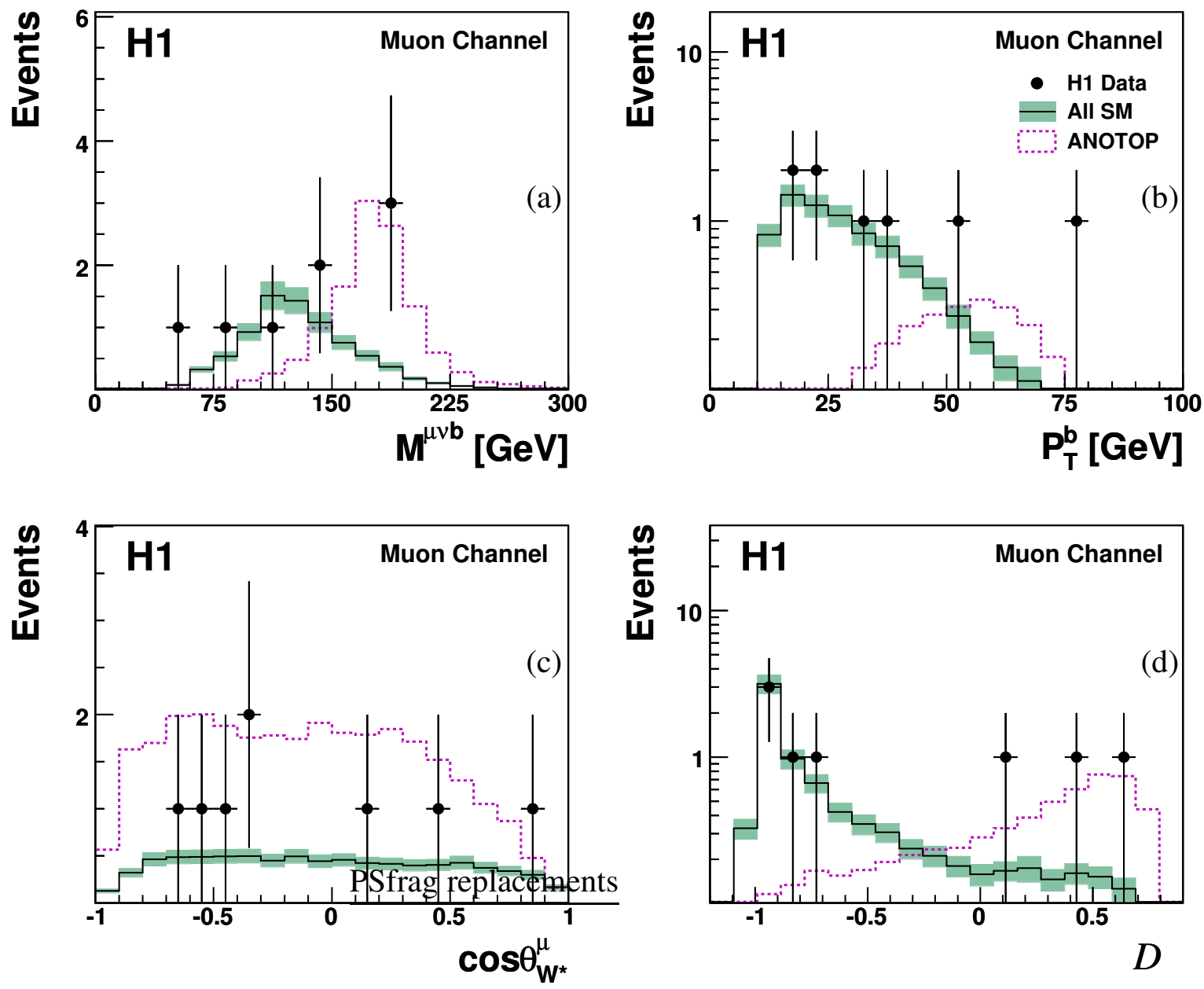

Figure 4: Distributions of observables in the muon channel used to differentiate single top production from SM background processes in the top preselection. Shown are the reconstructed top mass $M^{\mu \nu b}$ (a), the transverse momentum of the reconstructed $b$-jet candidate $P_{T}^{b}(\mathrm{~b})$, the $W$ decay angle $\cos \theta_{W^{*}}^{\mu}$ (c), and the combination of these observables into a MLP-based discriminant $D$ (d). The data are shown as points, the total SM expectation as the open histogram with systematic and statistical uncertainties added in quadrature (shaded band). Also shown is the signal prediction with arbitrary normalisation (dashed histogram). 


\section{Search for Single Top Quark Production at HERA (474 pb-1)}
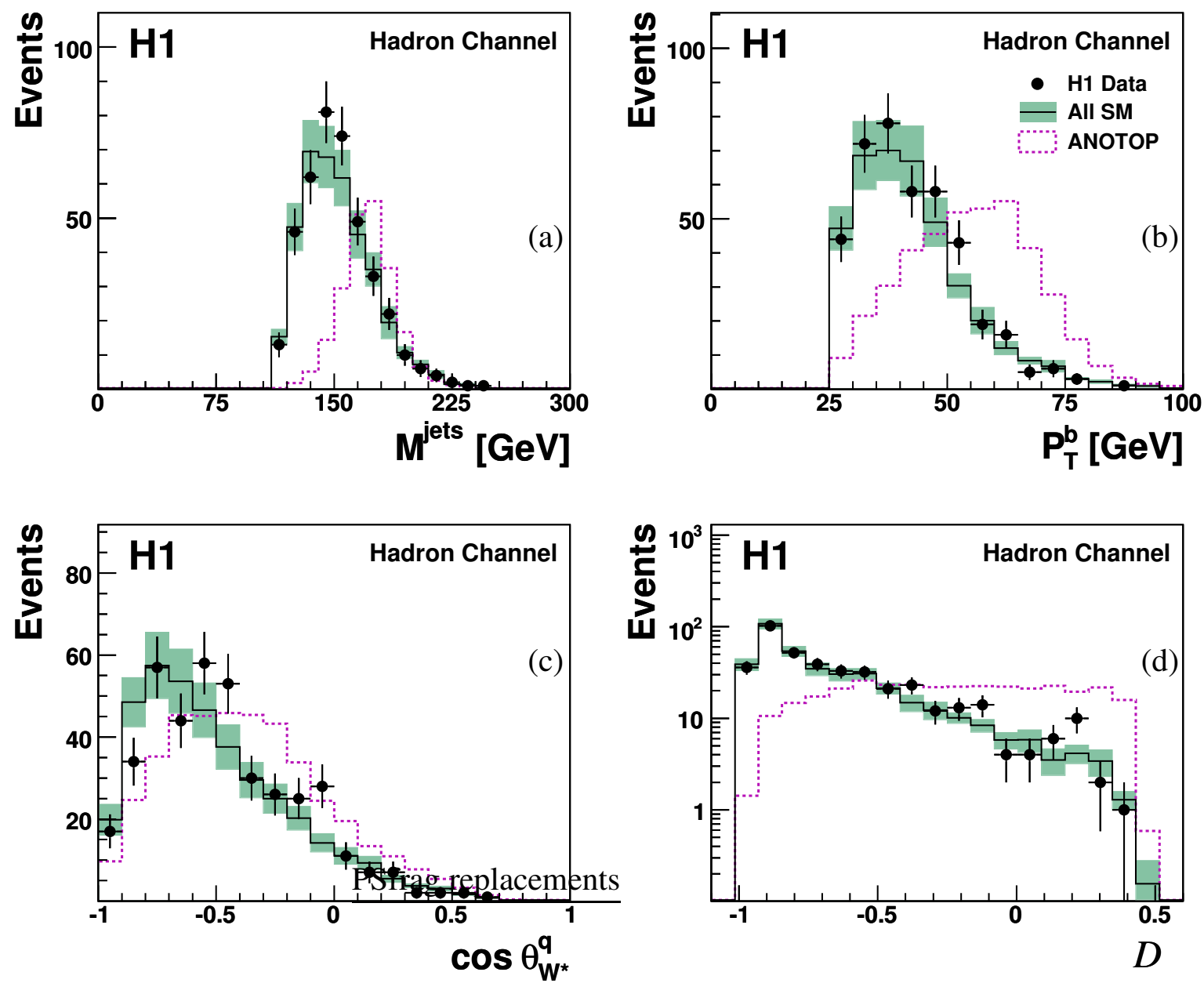

Figure 5: Distributions of observables in the hadronic channel used to differentiate single top production from SM background processes in the top preselection. Shown are the invariant mass of all jets $M^{\text {jets }}(\mathrm{a})$, the transverse momentum of the $b$-jet candidate $P_{T}^{b}(\mathrm{~b})$, the $W$ decay angle $\cos \theta_{W^{*}}^{q}$ (c), and the combination of these observables into a MLP-based discriminant $D$ (d). The data are shown as points, the total SM expectation as the open histogram with systematic and statistical uncertainties added in quadrature (shaded band). Also shown is the signal prediction with arbitrary normalisation (dashed histogram). 


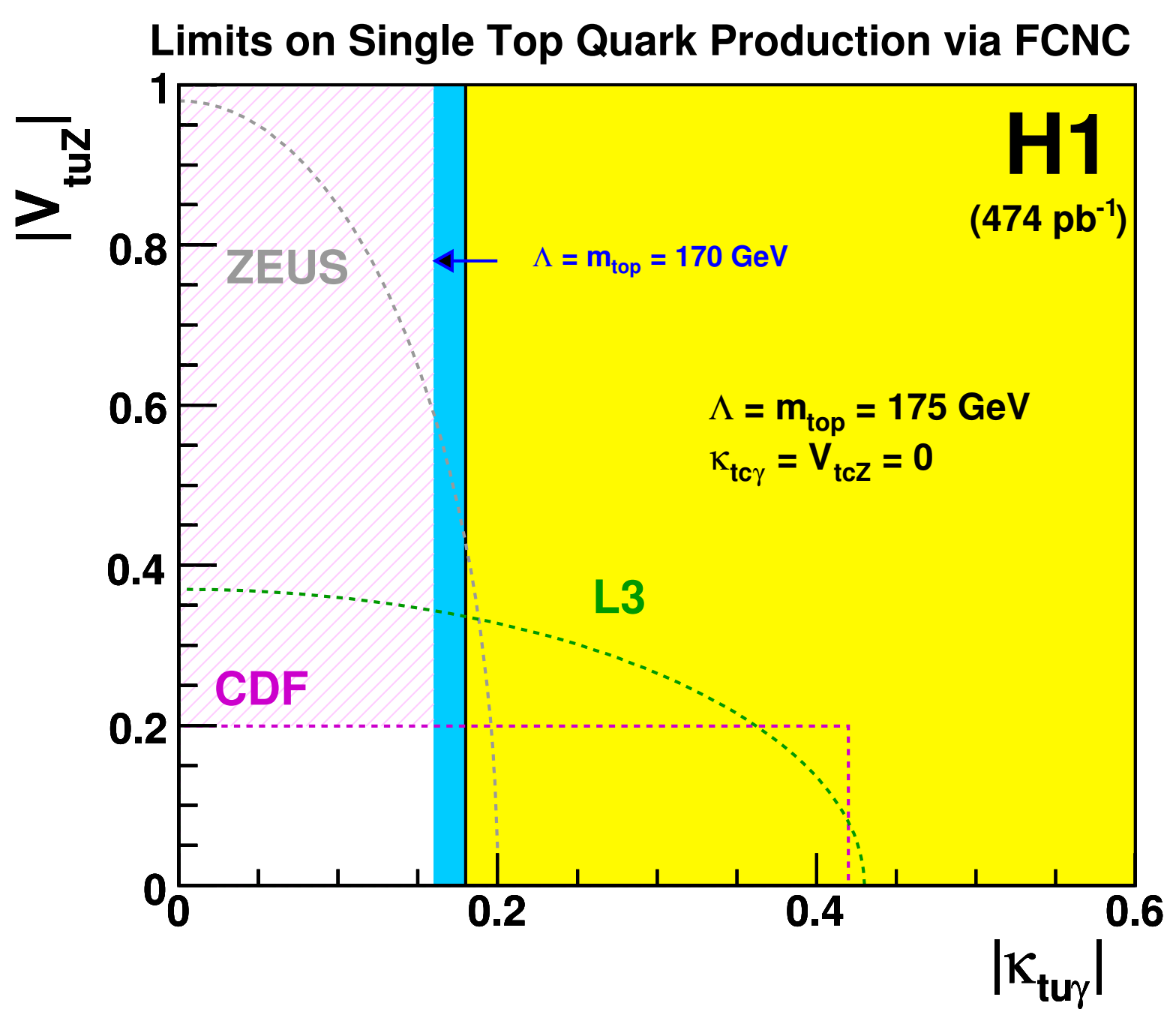

Figure 6: Upper limits at $95 \% \mathrm{CL}$ on the anomalous $\kappa_{t u \gamma}$ and $V_{t u Z}$ couplings obtained assuming $\Lambda \equiv m_{\text {top }} \equiv 175 \mathrm{GeV}$. Anomalous couplings of the charm quark are neglected: $\kappa_{t c \gamma}=V_{t c Z}=0$. The domain excluded by $\mathrm{H} 1$ is represented by the light shaded area. The dark shaded band shows the region additionally excluded if $\Lambda \equiv m_{\text {top }}$ is varied to $170 \mathrm{GeV}$. The hatched area corresponds to the domain excluded at the Tevatron by the CDF experiment $[9,10]$. Also shown are limits obtained at LEP by the L3 experiment [42] and at HERA by the ZEUS experiment [43]. 